\title{
WISP2 exhibits its potential antitumor activity via targeting ERK and E-cadherin pathways in esophageal cancer cells
}

\author{
Da-Min Chai ${ }^{1 \dagger}$, Yan-Zi Qin ${ }^{1 \dagger}$, Shi-Wu Wu', Li Ma', Yuan-Yuan Tan', Xiang Yong ${ }^{1}$, Xiao-Li Wang ${ }^{1}$, \\ Z. Peter Wang ${ }^{2,3^{*}}$ and Yi-Sheng Tao ${ }^{{ }^{*}}$
}

\begin{abstract}
Backgrounds: Emerging evidence has demonstrated that WISP2 is critically involved in cell proliferation, migration, invasion and metastasis in cancers. However, the function of WISP2 in esophageal squamous cell carcinoma (ESCC) is largely unclear. Therefore, we aim to explore the effects and the potential mechanism of WISP2 on proliferation and motility and invasion of ESCC cells.

Methods: Cell proliferation was detected by MTT assay and apoptosis was measured by FACS in ESCC cells after WISP2 downregulation and overexpression. Cell migration and invasion were analyzed by wound healing assay and transwell migration assay, respectively. The expression of ERK-1/2, Slug and E-cadherin was measured by Western blot respectively. IHC was performed to measure the expression of WISP2 in ESCC tissues.

Results: WISP2 overexpression is associated with survival in ESCC patients. WISP2 overexpression inhibited cell growth and induced cell apoptosis, suppressed cell migration and invasion in ESCC cells. Moreover, WISP overexpression retarded tumor growth in mouse model. WISP2 downregulation enhanced cell growth, inhibited apoptosis, promoted cell migration and invasion in ESCC cells. Mechanistically, WISP2 exerts its tumor suppressive functions via regulation of ERK1/2, Slug, and E-cadherin in ESCC cells.

Conclusions: Our findings suggest that activation of WISP2 could be a useful therapeutic strategy for the treatment of ESCC.
\end{abstract}

Keywords: Esophageal cancer, WISP2, Proliferation, Invasion, Migration

\section{Background}

Esophageal cancer is the common malignant neoplasm, which arises from the mucosa or gland of the esophagus. An estimated 17,290 patients will be diagnosed with esophageal cancer, and 15,850 people will die due to this disease in 2018 in the United States [1]. The reasons of esophageal cancer development are unclear. It has been reported that dietary habits, environmental factors, stress, and genetic mutations could contribute to esophageal

\footnotetext{
*Correspondence: taoyishengbbyxy@163.com; zwang6@bidmc.harvard.edu ${ }^{\dagger}$ Da-Min Chai and Yan-Zi Qin contributed equally to this work. ${ }^{2}$ Department of Biochemistry and Molecular Biology, School of Laboratory Medicine, Bengbu Medical College, Anhui 233030, China

'Department of Pathology, the First Affiliated Hospital of Bengbu Medical University, Bengbu Medical College, Changhuai road 287\#, Bengbu, Anhui 233000, People's Republic of China

Full list of author information is available at the end of the article
}

cancer [2]. The current treatments of esophageal cancer include surgical resection, chemotherapy, radiotherapy, or combined strategy $[3,4]$. Due to the metastatic incidence at diagnosis, drug resistance after chemotherapy and recurrence after surgery, long-term survival is still low in patients with esophageal cancer [5]. Early detection and treatment of esophageal cancer is a promising strategy to improve the survival rate in esophageal cancer patients. However, esophageal cancer patients often exhibit the onset of symptoms at diagnosis, which leads to poor prognosis. Alternatively, identification of molecular mechanism underlying esophageal tumorigenesis could be helpful for the better treatment of esophageal cancer patients.

WISP2 (WNT1 inducible signaling pathway protein 2) was reported to be upregulated in the mammary epithelial cells transformed by the Wnt-1 oncogene [6]. Subsequently, 
WISP2 was found to be directly regulated by the estrogen receptor in human breast cancer cells [7]. One study further identified that WISP2 expression was enhanced by serum and correlated with serum-induced cell proliferation in breast cancer cells, demonstrating that WISP2 could enhance cell proliferation in breast cancer [8]. Moreover, WISP2 waas reported to have higher expression in breast cancer patients at late stages with metastasis, indicating that WISP2 plays an oncogenic role in breast cancer [9]. Controversially, several studies revealed that WISP2 is a tumor suppressor in breast cancer [10, 11]. For example, one group observed that WISP2 level is inversely correlated with lymph node positivity. Notably, WISP2 was a negative regulator of migration and invasion via regulation of MMP-2 (matrix metalloproteinase-2), and MMP-9 in breast cancer cells [10]. Consistently, WISP2 overexpression led to cell cycle arrest at G1/G1 phase, inhibited cell growth, suppressed tumor growth in the xenograft model in breast cancer [11]. Additionally, WISP2 downregulation triggered EMT in breast cancer cells, while WISP2 overexpression suppressed cell proliferative and invasive phenotypes in breast cancer cells [12]. In gastric cancer patients, WISP2 expression is associated with tumor stage, differentiation status, and overall survival [13]. Another group reported that WISP2 was highly expressed in gastric tissues compared to adjacent normal tissues [14]. It has been reported that lower WISP2 expression was observed in pancreatic cancer tissues [15]. These studies indicated that WISP2 plays an important role in the development and progression of human cancers.

Emerging evidence has shown that WISP2 is critically involved in tumorigenesis in various types of human cancers. However, there are no available reports to show the function of WISP2 in ESCC. Therefore, in the current study, we explored the functions of WISP2 in ESCC cells via upregulation and downregulation of WISP2, including cell growth, apoptosis, migration and invasion. Moreover, we determined mechanisms of WISP2-invloved in ESCC progression. Our study will provide the direct evidence for role of WISP2 in ESCC.

\section{Materials and methods}

\section{Human ESCC samples}

The 216 cases of paraffin-embedded ESCC, 60 cases of normal esophageal epithelium, and 20 cases of esophagitis were collected at the time of surgery between 2006 and 2008 at the first affiliated hospital to Bengbu Medical College (Anhui, China). The clinical information of patients is described in detail in Table 1. ESCC patients were treated with surgery, but not with chemotherapy or radiation therapy. The 28 cases of ESCC specimens were frozen in liquid nitrogen immediately and stored at -80 ${ }^{\circ} \mathrm{C}$. The study was approved by the Ethics Committee of Bengbu Medical College.
Table 1 Expression of WISP2 in ESCC and the relationship with clinicopathological parameters.

\begin{tabular}{|c|c|c|c|c|c|c|}
\hline \multirow[t]{2}{*}{ Parameter } & \multirow[t]{2}{*}{ cases } & \multicolumn{3}{|c|}{ WISP2 } & \multirow[t]{2}{*}{$x^{2}$} & \multirow[t]{2}{*}{$P$} \\
\hline & & + & - & Positive(\%) & & \\
\hline \multicolumn{7}{|l|}{ Gender } \\
\hline Male & 121 & 44 & 77 & 36.36 & & \\
\hline Female & 95 & 33 & 62 & 34.74 & 0.06 & 0.89 \\
\hline \multicolumn{7}{|l|}{ Age(years) } \\
\hline$\leq 60$ & 86 & 35 & 51 & 40.70 & & \\
\hline$>60$ & 130 & 42 & 88 & 32.31 & 0.21 & 0.25 \\
\hline \multicolumn{7}{|l|}{ Tumor grade } \\
\hline 1 & 50 & 20 & 30 & 40.00 & & \\
\hline$\|$ & 78 & 29 & 49 & 37.18 & & \\
\hline III & 88 & 28 & 60 & 31.82 & 1.78 & 0.62 \\
\hline \multicolumn{7}{|l|}{ Infiltration depth } \\
\hline Under serous layer & 99 & 71 & 28 & 71.72 & & \\
\hline Serous layer & 117 & 6 & 111 & 0.51 & 103.65 & $<0.01$ \\
\hline \multicolumn{7}{|l|}{ Lymph node metastasis } \\
\hline Yes & 112 & 2 & 110 & 1.79 & & \\
\hline No & 104 & 75 & 29 & 72.12 & 116.27 & $<0.01$ \\
\hline \multicolumn{7}{|l|}{ TNM stages } \\
\hline I,Il stages & 88 & 71 & 17 & 80.68 & & \\
\hline III,IV stages & 128 & 6 & 122 & 4.69 & 131.28 & $<0.01$ \\
\hline \multicolumn{7}{|l|}{ Diameter(cm) } \\
\hline$<4$ & 97 & 36 & 61 & 37.11 & & \\
\hline$\geq 4$ & 119 & 41 & 78 & 34.45 & 0.69 & 0.78 \\
\hline \multicolumn{7}{|l|}{ General type } \\
\hline Ulcerative type & 67 & 21 & 46 & 23.8 & & \\
\hline Medullary type & 97 & 34 & 63 & 23.8 & & \\
\hline Mushroom type & 41 & 18 & 23 & 18.2 & & \\
\hline Constrictive type & 11 & 4 & 7 & 25.0 & 0.33 & 0.96 \\
\hline \multicolumn{7}{|l|}{ Location } \\
\hline Top & 28 & 14 & 14 & 50.00 & & \\
\hline Middle & 116 & 34 & 82 & 29.31 & & \\
\hline Bottom & 72 & 29 & 43 & 40.28 & 5.22 & 0.07 \\
\hline
\end{tabular}

Table 2 Expression of WISP2 in normal esophageal mucosa and ESCC

\begin{tabular}{lrlllll}
\hline Parameter & Total & \multicolumn{3}{l}{ WISP2 expression } & $X^{2}$ & $P$ value \\
\cline { 3 - 6 } & & + & - & Positive (\%) & & \\
\hline Normal mucosa & 60 & 31 & 29 & 51.66 & 5.78 & 0.02 \\
Tumor tissue & 216 & 77 & 139 & 35.65 & & \\
\hline
\end{tabular}




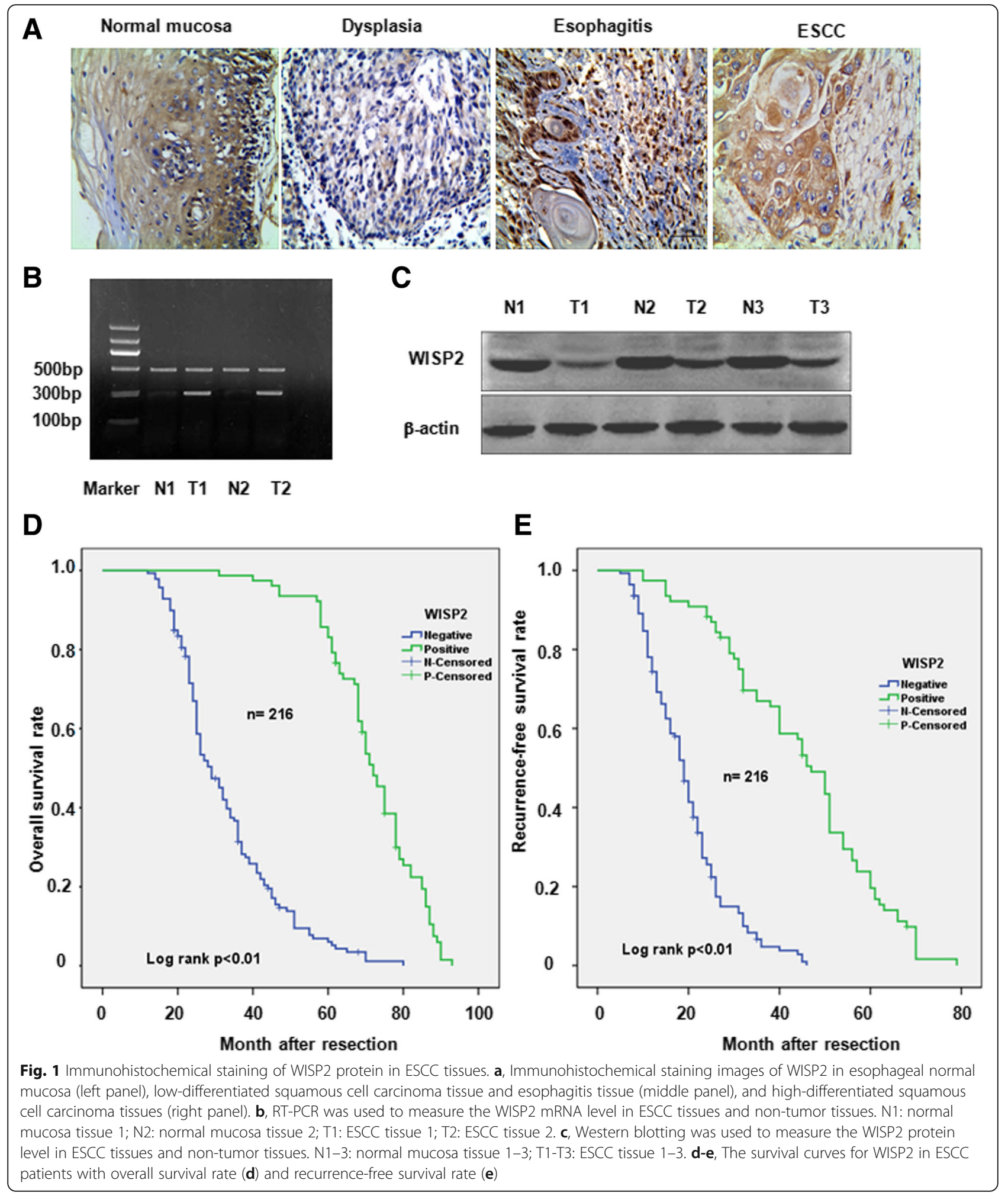


Table 3 Expression of WISP2 mRNA in normal esophageal mucosa and ESCC

\begin{tabular}{|c|c|c|c|}
\hline Parameter & Cases & $\bar{x} \pm s$ & $t$ \\
\hline Normal mucosa & 28 & $0.830 \pm 0.027$ & $-17.161<0.01$ \\
\hline Tumor tissue & 28 & $0.452 \pm 0.114$ & \\
\hline
\end{tabular}

\section{Histological sections and immunohistochemistry}

Immunohistochemical studies were performed to determine the expression of WISP2 in tumors as described before [16].

\section{Cell culture}

Human ESCC cell lines, EC9706 and Eca109 cells, were purchased from the Cell Resource Center, Shanghai Institutes for Biological Sciences (Shanghai, China) and cultured in Dulbecco's modified Eagle's medium (DMEM) containing $10 \%$ fetal bovine serum (FBS, Sigma, USA). Subcultures were prepared using $0.05 \%$ trypsin solution (Invitrogen, Carlsbad) and seeded in 6- or 96-well tissue culture plates.

\section{Transfection}

The transfection of WISP2 plasmid or WISP2 siRNA or control siRNA was conducted using Lipofectamine 2000 reagent (Invitrogen, Carlsbad, CA, USA). All WISP2 siRNAs were purchased from shanghai GenePharma in China.

\section{Real-time quantitative PCR}

The total RNA of ESCC transfected cells in each group was isolated with Trizol (Invitrogen, Carlsbad) and reverse-transcribed to cDNA with Reverse Transcription System [17]. Quantitative real-time PCR was performed on an ABI 7900 System with SYBR green (Takara, China). Primers of WISP2 were as follows: TGC TGC CCT GAG TGG GTG (forward, 5'-3') and GAA GCG GTT CTG GTT GGA C (reverse, $5^{\prime}-3^{\prime}$ ). Transcripts were quantified with GAPDH (glyceraldehyde 3-phosphate dehydrogenase) as an internal standard.

\section{Cell viability assay}

The ESCC cells were seeded in 6-well plates overnight and transfected with WISP2 plasmid or its siRNA, for $48 \mathrm{~h}$. Then, ESCC cells were trypsinizated and seeded in 96 well plates for $72 \mathrm{~h}$. Cell viability was detected by MTT assay as described previously [18].

Table 4 The statistics and univariate analysis of the patients with ESCC

\begin{tabular}{lrcll}
\hline Parameter & Cases & 5-y survival rate (\%) & $X^{2}$ & $P$ \\
\hline WISP2 & & & 146.25 & $<0.01$ \\
+ & 77 & 85.7 & & \\
- & 139 & 5.76 & & \\
\hline
\end{tabular}

\section{Apoptotic cells measurement}

Apoptotic cell death was detected using the annexin V-FITC / propidium iodide (PI) staining kit (BestBio, Shanghai, China) [19]. Briefly, after transfection with WISP2 plasmid or siRNAs in ESCC cells in different groups, the cells were harvested, washed with PBS and resuspended in the binding buffer provided by the kit. The cells were then labeled with annexinV- FITC/ PI and the percentage of apoptosis cells was quantified using the Multicycle AV software (FACSAria, BD Biosciences, CA, USA).

\section{Western blotting}

Western blotting was performed according to the established methods $[20,21]$. The transfected ESCC cells were rinsed twice with ice-cold phosphate buffered saline (PBS, $\mathrm{pH}$ 7.4), lysed in a cooled buffer, and sonicated for $5 \mathrm{~s}$ on ice. Then the lysates were boiled in a water bath for 10 $\mathrm{min}$, and centrifuged at $12,000 \mathrm{~g}$ for $10 \mathrm{~min}$ at $4{ }^{\circ} \mathrm{C}$. The protein concentrations in the supernatant were measured by BCA kit (Pierce, USA). Equal amounts of proteins were separated by $10 \%$ SDS-polyacrylamide gel electrophoresis (SDS-PAGE) and transferred to nitrocellulose membranes (Amersham, Germany).The protein expression was measured as described previously $[20,21]$.

\section{Transwell invasion assay}

The migration and invasion assays of transfected ESCC cells were performed using Transwell cell-culture chambers (Corning, USA). For invasion assay, the chamber was coated with the admixture of Matrigel (BD, USA). The treated cells were seeded into the upper chamber with serum-free medium $\left(5 \times 10^{4}\right.$ cells), and the bottom chamber contained DMEM medium with $10 \%$ FBS. When the cells invaded for $20 \mathrm{~h}$, the cells were washed, fixed, stained with Calcein-AM for 20 min or crystal violet. Then the invaded cells were counted and taken pictures under microscope (Hanrong Company, Shanghai).

\section{In vivo experiments}

The animal experiments were operated following the previous description [22]. To establish all the nude mice models of ESCC which were divided into three groups, Eca109 control group, Eca109-empty vector group, Eca109-WISP2 expression. We also injected Eca109 cells with control siRNA or WISP2 siRNA transfection to nude mice. To choose the right axillary injection amount of cell suspension, regular measurement of tumor growth in volume, record and process data, and draw the tumor growth curve. After 28 days, the nude mice were sacrificed by cervical dislocation, and tumor tissues were taken out. Tumor weights were measured. The expression of WISP2, E-cadherin, ERK1/2, and Slug was measured by Western blotting analysis. 


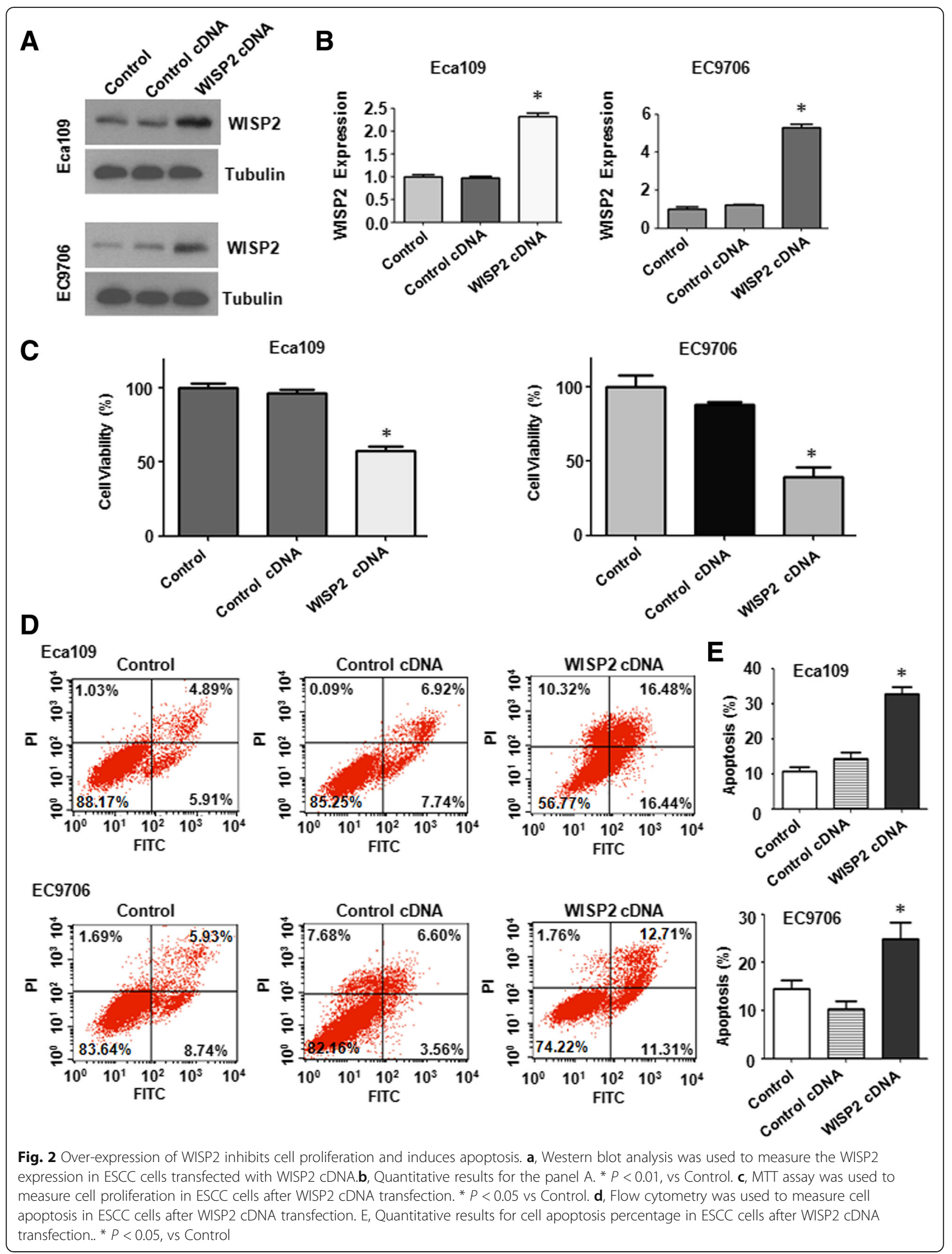




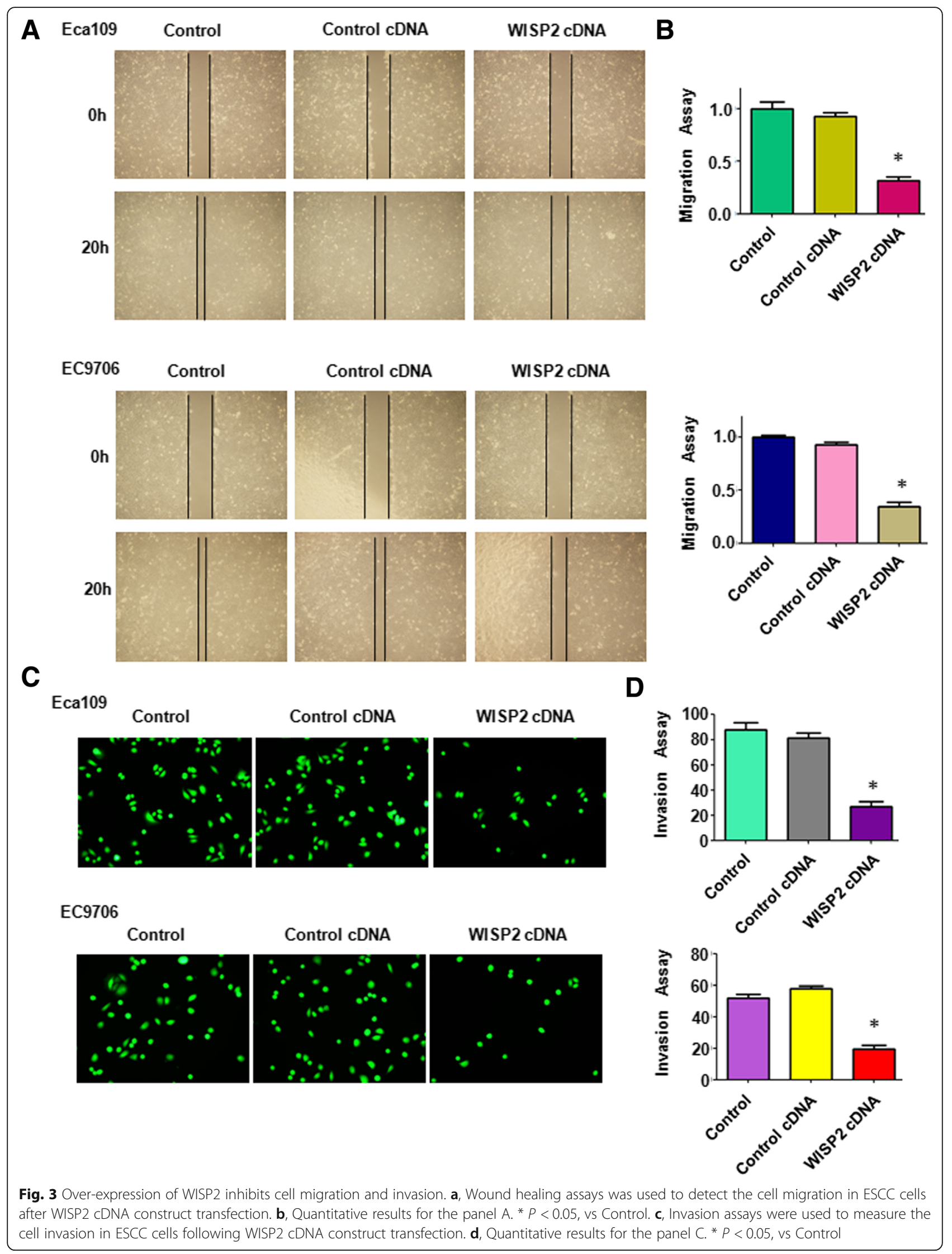




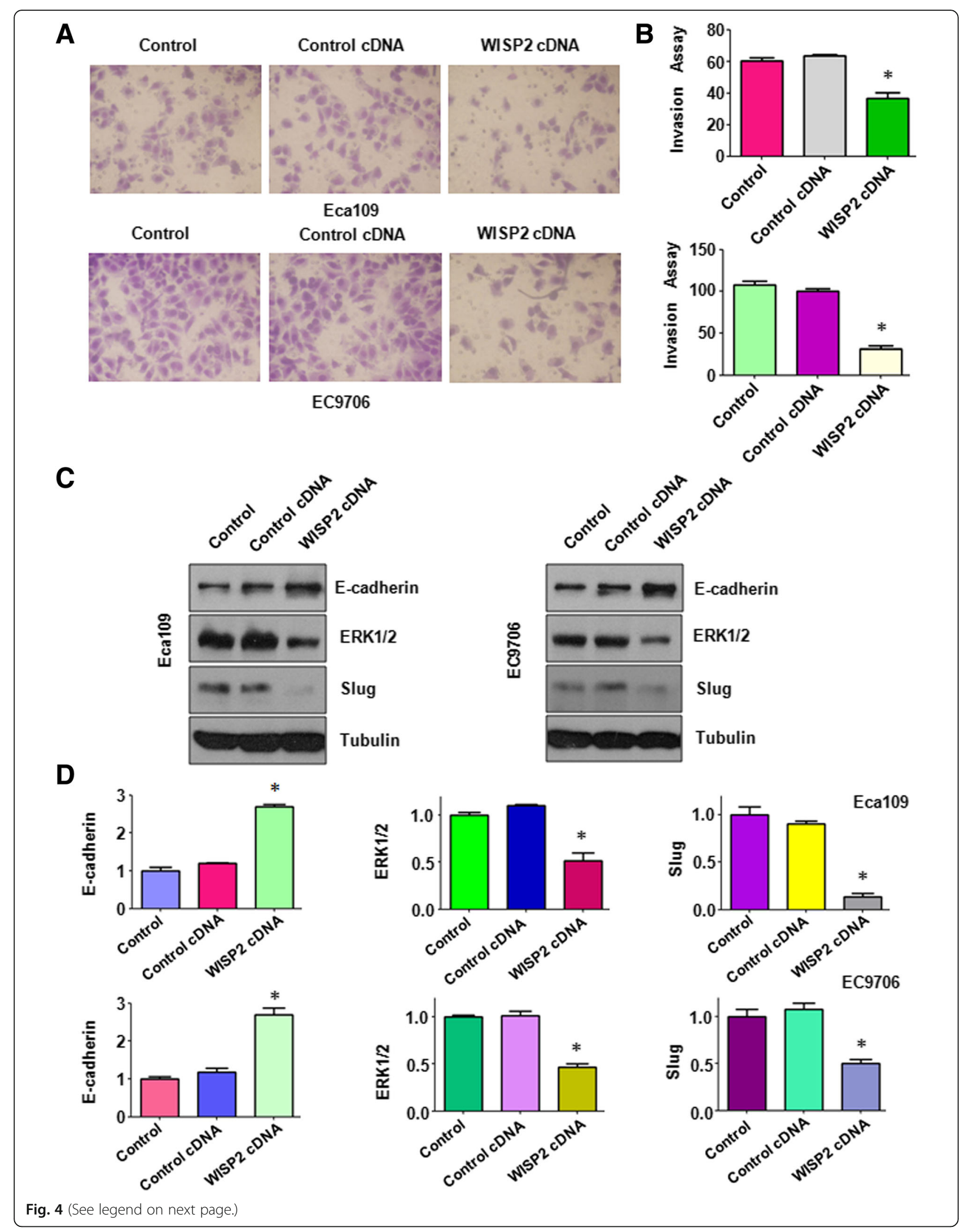


(See figure on previous page.)

Fig. 4 Over-expression of WISP2 regulates E-cadherin, ERK1/2, and Slug. a, Invasion assays were used to measure the cell invasion in ESCC cells following WISP2 cDNA construct transfection. b, Quantitative results for the panel A. ${ }^{*} P<0.05$, vs Control. c, Western blot analysis was used to measure the expression of E-cadherin, ERK1/2, and Slug in ESCC cells transfected with WISP2 CDNA. d, Quantitative results for the panel C. ${ }^{*} P<0.05$, vs Control

\section{Statistical analysis}

All data were expressed as mean $\pm \mathrm{SD}$. Comparisons between groups were performed using one-way ANOVA followed by Tukey's post-hoc test. Differences were evaluated using Student's t test. Statistical significance was indicated as $p$-value $<0.05$. Statistical tests were performed with GraphPad Prism version 5 for Windows (GraphPad Software, San Diego, California, USA).

\section{Results}

Expression of WISP2 is associated with clinical significance in ESCC

To explore the association of WISP2 and clinicopathologic significance in ESCC, we measured the protein of WISP2 in 216 cases of ESCC and 60 cases of normal esophageal epithelium by immunohistochemistry method (Table 1). We found that the positive expression rate of WISP2 protein was 35.65\% (77/216) in 216 cases of ESCC tissues. $64.35 \%$ tumor tissues have negative expression of WISP2, suggesting that lower expression of WISP2 was exhibited in ESCC tissues. $51.66 \%$ of positive expression of WISP2 in 60 cases of esophageal normal mucosa was observed (Table 2). Compared with the positive expression rate of WISP2 in esophageal normal mucosa tissues (Fig. 1A left panel) esophageal dysplasia tissues (Fig. 1A middle left panel), and esophagitis tissue (Fig. 1A middle right panel), WISP2 level in ESCC tissues was downregulated (Fig. 1A right panel). The expression of WISP2 was associated with tumor lymph node metastasis, depth of tumor infiltration, histological differentiation and TNM stage (Table 1), but was not associated with the patient's gender, age, tumor location, the general type, and diameter (Table 1). To further validate our result, the mRNA of WISP2 was detected by Reverse Transcription PCR (RT-PCR) in 28 cases of ESCC and adjacent normal tissues. We found that there was a significant difference for WISP2 mRNA level between adjacent normal tissues and ESCC tumors (Fig. $1 \mathrm{~B}$ and Table 3). Our western blotting results showed down-regulation of WISP2 in ESCC tumor tissues compared with adjacent normal tissues (Fig. 1C). Furthermore, we investigated the relationship between WISP2 and survival in 93 cases of ESCC. Our Kaplan-Meier curves analysis (log-rank test) showed that there was a significance difference between the positive expression of WISP2 groups and the negative expression of WISP2 groups in overall survival rate (Fig. 1D and Table 4) and recurrence-free survival rate (Fig. 1E). Taken together, lower expression of WISP2 in ESCC tissues was associated with lymph node metastasis, depth of invasion and the stage.

\section{Overexpression of WISP2 inhibits cell growth and induces apoptosis}

In order to explore the role of WISP2 in ESCC, the plasmid with WISP2 cDNA was transfected into ESCC cells. The efficacy of WISP2 cDNA transfection for overexpression of WISP2 in ESCC cells was validated by Western blotting analysis. Our result showed that WISP2 was significantly overexpressed in ESCC cells after cDNA transfection (Fig. 2A and B). MTT assay was used to measure cell growth in WISP2-overexpressing ESCC cells. We found that overexpression of WISP2 led to $45 \%$ cell growth inhibition in Eca109 cells $(p=0.007)$ and 55\% growth inhibition in EC9706 cell $(p=0.002)$ compared with control cDNA transfection group (Fig. $2 \mathrm{C})$. To further characrized the function of WISP2 in ESCC cells, we measured the cell apoptotic death by Annexin V-FITC/PI method in ESCC cells after WISP2 overexpression. We found that upregulation of WISP2 increased the percentages of apoptotic cells from $14.56 \%$ in control cDNA transfection group to $32.92 \%$ in Eca109 cells with WISP2 cDNA transfection $(p=0.002)$, and from $10.16 \%$ in control cDNA group to $24.02 \%$ in EC9706 $(p=0.012)$ cell line (Fig. 2D and E). This data implied that WISP2 suppressed cell growth partly due to induction of apoptosis in ESCC cells.

\section{Overexpression of WISP2 retards cell migration and invasion}

Next, we examined whether WISP2 could regulate cell migration and invasion in ESCC cells. Wound healing assay was performed to detect the migration of ESCC cells after WISP2 overexpression. We found that up-regulation of WISP2 inhibited 60 to $70 \%$ cell migration in Eca109 cells $(p=0.009)$ and EC9706 $(p=0.002)$ cell lines (Fig. 3A and B). Our matrigel invasion assay results showed that overexpression of WISP2 remarkably retarded 65 to $70 \%$ cell invasion in Eca109 cells ( $p=$ 0.002) and EC9706 ( $p=0.007)$ cell lines (Fig. 3C-3D). Similarly, the invaded cells with WISP2 overexpression that stained with crystal violet also were reduced to $50 \%$ in Eca109 cells $(p=0.0035)$ and $30 \%$ cell invasion in EC9706 ( $p=0.0016)$ cell lines, respectively (Fig. 4A-4B). Our findings indicate that WISP2 overexpression retarded cell migration and invasion in ESCC cells. 


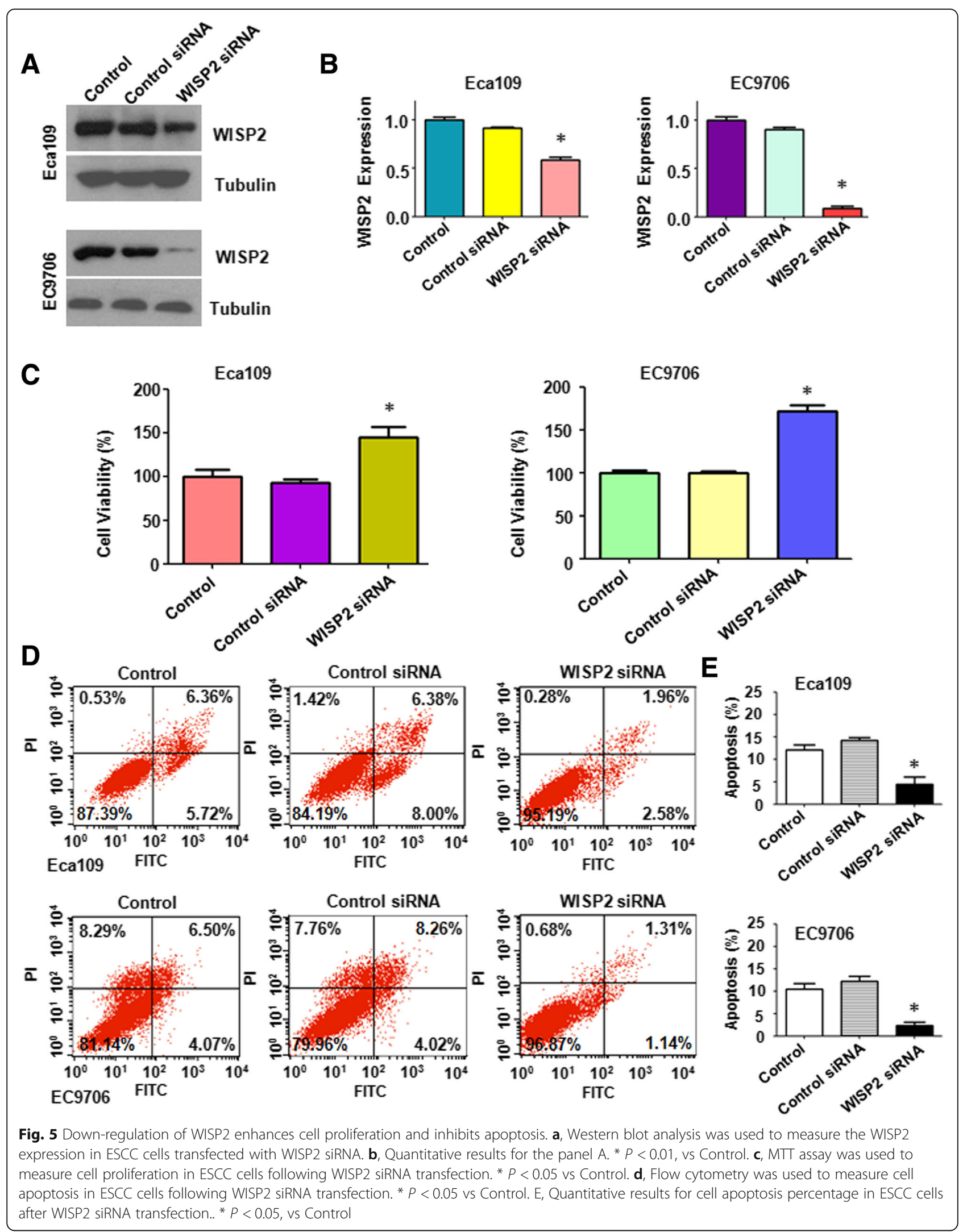




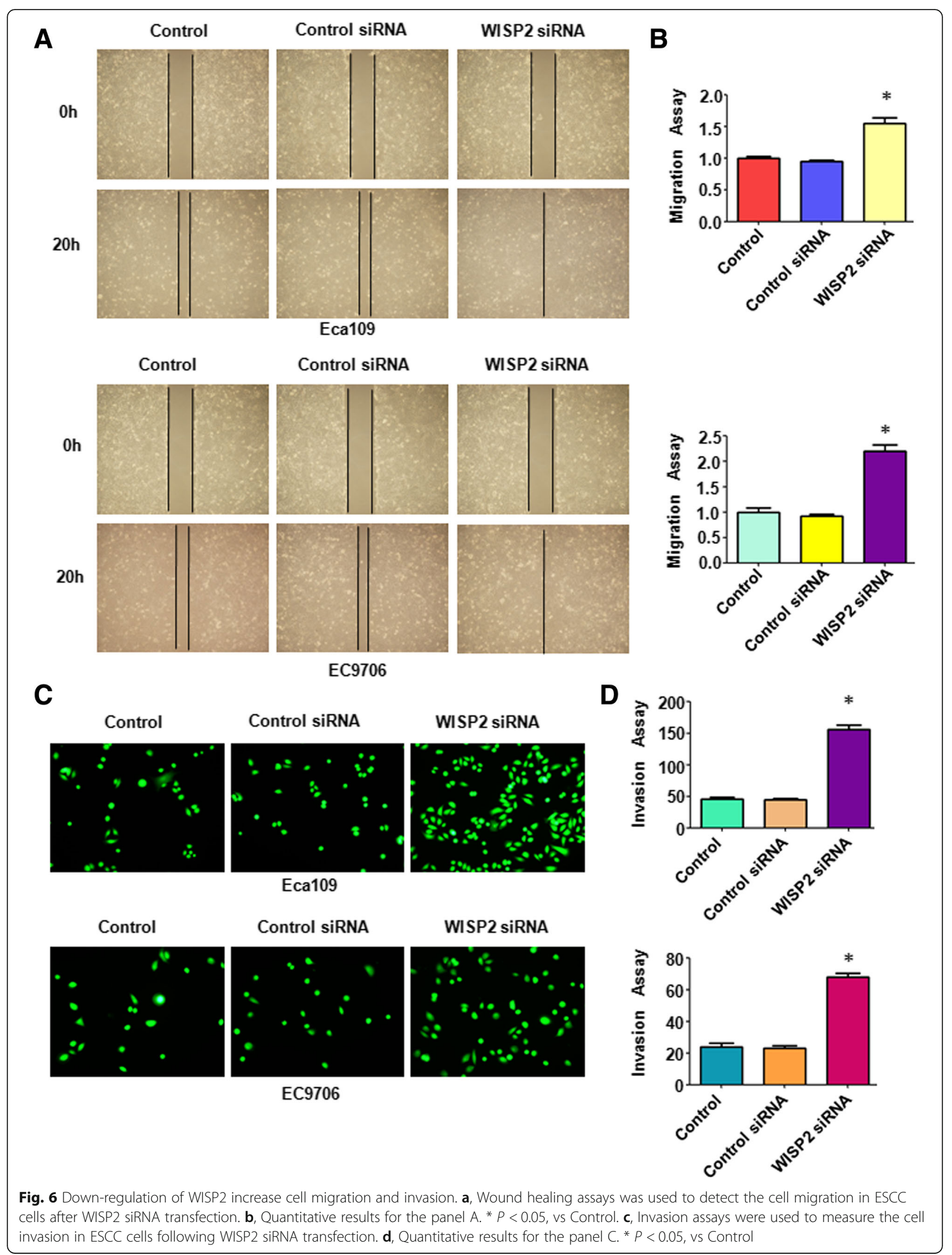




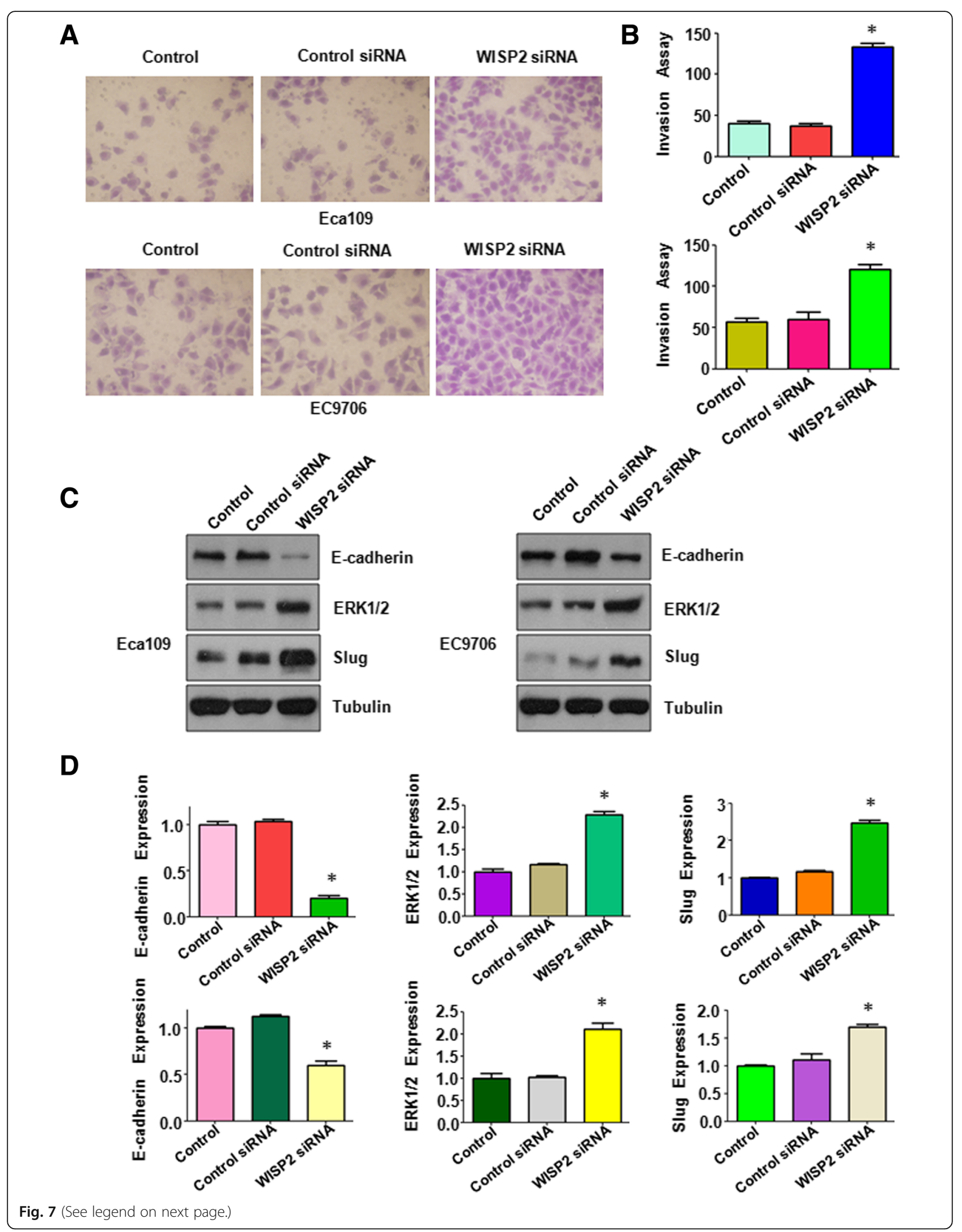


(See figure on previous page.)

Fig. 7 Down-regulation of WISP2 regulates E-cadherin, ERK1/2, and Slug. a, Invasion assays were used to measure the cell invasion in ESCC cells following WISP2 siRNA transfection. $\mathbf{b}$, Quantitative results for the panel A. ${ }^{*} P<0.05$, vs Control. $\mathbf{c}$, Western blot analysis was used to measure the expression of E-cadherin, ERK1/2, and Slug in ESCC cells transfected with WISP2 siRNA. d, Quantitative results for the panel C. ${ }^{*} P<0.05$, vs Control

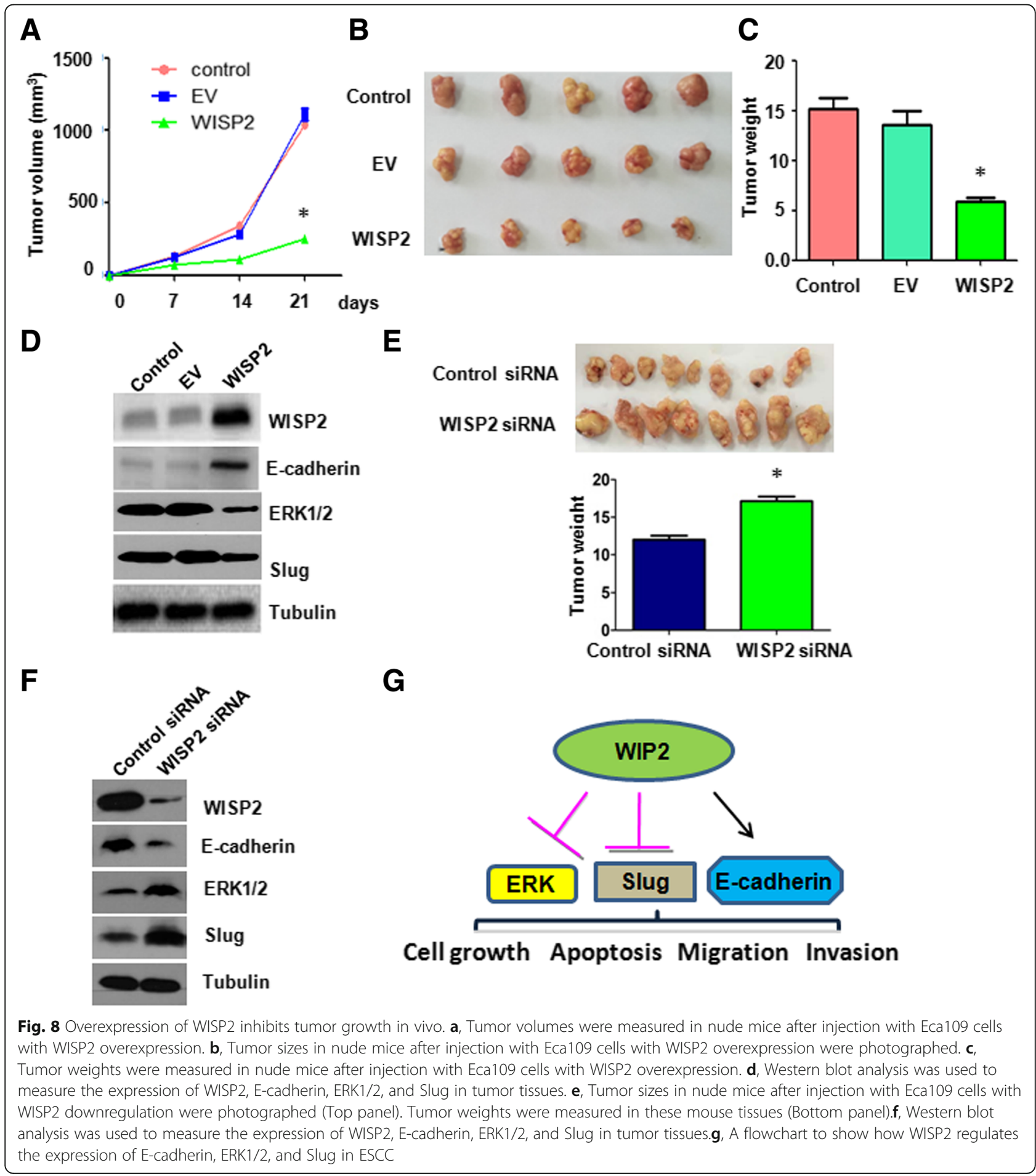




\section{Overexpression of WISP2 decreases ERK expression and increases E-cadherin level}

To further determine the mechanism of WISP2-mediated tumor suppression, we investigated whether overexpression of WISP2 could regulate ERK, Slug, and E-cadherin in ESCC cells. Our Western blotting data showed that E-cadherin level was dramatically upregulated in the WISP2 cDNA transfected cells (Fig. $4 \mathrm{C}$ and D). We also observed that the expression of ERK and Slug was downregulated in cells after WISP2 cDNA transfection in ESCC cells (Fig. 4C and D). These data indicated that overexpression of WISP2 exerted its tumor suppressive function partly via inhibition of ERKand Slug, but upregulation of E-cadherin in ESCC cells.

\section{Downregulation of WISP2 enhances cell growth and inhibits apoptosis}

To further validate the function of WISP2, ESCC cells were transfected with WISP2 siRNA or control siRNA. The efficacy of WISP2 siRNA transfection was measured by Western blotting. Our result showed that WISP2 siRNA transfection significantly reduced the expression of WISP2 in both ESCC cell lines (Fig. 5A and B). Cell growth was determined by MTT in ESCC cells after WISP2 siRNA transfection. We found that down-regulation of WISP2 promoted 50\% cell growth in Eca109 cells $(p=0.0027)$ and $70 \%$ cell growth in EC9706 cell $(p=0.0004)$ compared with control siRNA transfection group (Fig. 5C). In line with this, we found that down-regulation of WISP2 decreased the percentages of apoptotic cells from $14.38 \%$ in control siRNA transfection group to $4.54 \%$ in Eca109 cells with WISP2 siRNA transfection $(p=0.0028)$, and from $12.28 \%$ in control siRNA group to $2.45 \%$ in EC9706 $(p=0.0012)$ cell line (Fig. 5D). These findings indicate the tumor suppressive role of WISP2 in ESCC cells.

\section{Downregulation of WISP2 promotes cell migration and invasion}

To further dissect the role of WISP2 in cell migration and invasion, wound healing assay was applied for detecting cell migration in cells after downregulation of WISP2 in ESCC cells. Our wound healing assay results showed that WISP2 siRNA transfection led to an increase of cell wound healing in Eca109 cells $(p=0.0007)$ and EC9706 $(p=0.0003)$ cells (Fig. 6A and B). Moreover, Transwell chamber assay was conducted to measure the cell invasion in ESCC cells after WISP2 siRNA transfection. We found that WISP2 siRNA transfected cells showed 3-3.5 folds promotion of cell invasion in both Eca109 cells $(p<0.001)$ and EC9706 $(p=0.0011)$ compared to control siRNA transfected cells (Fig. 6C-D). In line with this, WISP2 downregulation led to a significant increase in cell invasion of Eca109 cells $(p=0.0288)$ and EC9706 $(\mathrm{p}<0.001)$ cells (Fig. 7A-B). Mechanistically, downregulation of WISP2 decreased E-cadherin level and increased the level of ERK1/2 and Slug in ESCC cells (Fig. 7C-D).

\section{Overexpression of WISP2 inhibits tumor growth in vivo}

The in vivo results showed that overexpression of WISP2 inhibited tumor growth using nude mouse model of ESCC (Fig. 8A). Tumor growth volume in WISP2 overexpression group was lower than control group and empty vector group (Fig. 8B). Consistently, the final tumor weight of WISP2 overexpressing stable cell group was lower than that of control groups (Fig. 8C). Furthermore, the expression of WISP2 and E-cadherin at protein level was increased in WISP2 cDNA transfection group, while the expression of ERK1/2 and Slug was decreased in WISP2 overexpressing group (Fig. 8D). Consistently, down-regulation of WISP2 retarded tumor growth in mice (Fig. 8E). Downregulation of WISP2 led to a decrease in E-cadherin expression and an increase in ERK1/2 and Slug level in mice tissues (Fig. 8F). Taken together, WISP2 could be a tumor suppressor via regulation of E-caherin, Slug, and ERK1/2 in ESCC (Fig. 8G).

\section{Discussion}

To date, the role of WISP2 in ESCC is unelucidated, although the function of WISP2 was investigated in a variety of human cancers $[14,23,24]$. In the present study, we reported that WISP2 overexpression inhibited cell growth and induced cell apoptosis, suppressed cell migration and invasion in ESCC cells. Moreover, WISP overexpression retarded tumor growth in mouse model. WISP2 downregulation enhanced cell growth, inhibited apoptosis, promoted cell migration and invasion in ESCC cells. Mechanistically, WISP2 exerts its tumor suppressive functions via regulation of ERK1/2, Slug, and E-cadherin in ESCC cells. Our study provides the new evidence showing WISP2 as a tumor suppressor in ESCC.

WISP2 has been reported to govern several gene expressions in human cancer cells. For instance, downregulation of WISP2 promoted PD-L1 level in breast cancer cells. Inactivation of PD-L1 restored the susceptibility of resistant cells with WISP2 downregulation to CTL treatment [25]. One study reported that WISP2 inhibited PI3K/Akt pathway and Skp2 expression, and subsequently increased p27 accumulation, induced FOXO3a expression and activity [11]. Further, loss of WISP2 enhanced cancer stem-like phenotype characterized and increased the level of stem cell markers Nanog and Oct $3 / 4$, and activated TGF- $\beta$ pathway in breast tumor cells [26]. WISP2 recombinant protein triggered MET (mesenchymal-epithelial transition) in pancreatic cancer cells [15]. HBx (hepatitis B virus X gene) mutants, frequently happened in HBV (hepatitis B virus)-related hepatocellular carcinoma, enhanced cell proliferation 
and migration via regulation of $\mathrm{Wnt} / \beta$-catenin signaling pathway [27]. HBx mutants stabilized $\beta$-catenin level via inhibition of GSK3 $\beta$ in HCC cells, resulted in increased WISP2 and c-Myc [27]. The expression level of WISP2 was enhanced in overexpressing TCF-4J (T-cell factor-4 isoform J) HCC cells, indicating that WISP2 could play a potential role in HCC progression [28].

WISP2 downregulation promoted cell growth, migration and invasion, but WISP2 overexpression suppressed cell metastasis through regulation of EMT and inhibition of MMP-9 and MMP-2 via ERK in gastric cancer cells [13]. It has been reported that WISP2 could regulate the Wnt/B-catenin signaling pathway in gastric cancer [14]. In line with these reports, our study dissected that WISP2 overexpression decreased expression of ERK1/2. WISP2 could inhibit the proliferation and induced apoptosis by suppressing ERK1/2 signal in ESCC. E-cadherin is a key molecule to be involved in EMT and cell invasion [29, 30]. WISP2 was reported to control migration and invasion via regulation of Snail and E-cadherin in breast cancer cells [10]. Our study also showed that WISP2 overexpression increased E-cadherin level and decreased Slug level in ESCC. Thus, WISP2 inhibit the invasion and migration by down-regulating slug and up-regulating E-cadherin expression in ESCC.

\section{Conclusions}

WISP2 plays a tumor suppressor role in ESCC cells. WISP2 overexpression inhibited cell growth and induced cell apoptosis, slowed cell migration and invasion in ESCC cells whereas downregulation of WISP2 has opposed effects. Mechanistically, WISP2 exerts its anti-tumor functions via regulation of ERK1/2, Slug, and E-cadherin in ESCC cells. Notably, WISP2 overexpression is associated with survival in ESCC patients. Our findings suggest that activation of WISP2 could be a potential therapeutic strategy for the treatment of ESCC. Without a doubt, further investigations are required to define the role of WISP2 using conditional knockout or knockin transgenic mouse model. The detailed molecular mechanisms how WISP2 is regulated and its downstream targets in ESCC are needed to be explored in the future.

\footnotetext{
Abbreviations

ERK: Extracellular signal-regulated kinase; ESCC: Esophageal squamous cell carcinoma; HBV: Hepatitis B virus; HBx: Hepatitis B virus X gene; MET: Mesenchymal-epithelial transition; MMP-2: Matrix metalloproteinase-2; PI3K: Phosphatidylinositol 3-kinase; Skp2: S-phase kinase associated protein 2; TCF-4J: T-cell factor-4 isoform J; WISP2: WNT1 inducible signaling pathway protein 2
}

\section{Acknowledgements}

Not applicable.

Availability of data and material

Please contact author for data requests.
Funding

Not applicable.

\section{Authors' contributions}

DMC, ZPW, and YST designed the study. DMC, YZQ, SWW performed the experiments. LM, YYT, XY, and XLW collected data and analyzed. DMC and SWW reviewed the histopathology and $I H C$ results. XY, XLW provided critical comments on the manuscript. All authors read and approved the final manuscript. DMC, ZPW, and YST wrote the manuscript.

\section{Ethics approval and consent to participate}

This study was approved by the Ethics and Research Committees of Bengbu Medical College, China. All animal experimental procedures were performed by following the China Animal Welfare Guidelines. The study protocol was approved by our Institutional Animal Care and Use Committee at Bengbu Medical College, Bengbu, China. All informed consents were obtained from individual participants included in this study.

\section{Consent for publication}

Not applicable.

\section{Competing interests}

The authors declare that they have no competing interests.

\section{Publisher's Note}

Springer Nature remains neutral with regard to jurisdictional claims in published maps and institutional affiliations.

\section{Author details}

${ }^{1}$ Department of Pathology, the First Affiliated Hospital of Bengbu Medical University, Bengbu Medical College, Changhuai road 287\#, Bengbu, Anhui 233000, People's Republic of China. ${ }^{2}$ Department of Biochemistry and Molecular Biology, School of Laboratory Medicine, Bengbu Medical College, Anhui 233030, China. ${ }^{3}$ Department of Pathology, Beth Israel Deaconess Medical Center, Harvard Medical School, 330 Brookline Ave, Boston, MA 02215, USA

Received: 17 November 2018 Accepted: 15 February 2019

Published online: 26 February 2019

\section{References}

1. Siegel RL, Miller KD, Jemal A. Cancer statistics, 2018. CA Cancer J Clin. 2018; 68:7-30.

2. Zulfigar $\mathrm{M}$, Bluth $\mathrm{MH}$, Bhalla A. Molecular diagnostics in esophageal and gastric neoplasms: 2018 update. Clin Lab Med. 2018;38:357-65.

3. Battaglin F, Naseem M, Puccini A, Lenz HJ. Molecular biomarkers in gastroesophageal cancer: recent developments, current trends and future directions. Cancer Cell Int. 2018;18(99).

4. Watanabe M, Okamura A, Toihata T, Yamashita K, Yuda M, Hayami M, et al. Recent progress in perioperative management of patients undergoing esophagectomy for esophageal cancer. Esophagus. 2018;15:160-4.

5. Barret M, Prat F. Diagnosis and treatment of superficial esophageal cancer. Ann Gastroenterol. 2018;31:256-65.

6. Pennica D, Swanson TA, Welsh JW, Roy MA, Lawrence DA, Lee J, et al. WISP genes are members of the connective tissue growth factor family that are up-regulated in wnt-1-transformed cells and aberrantly expressed in human colon tumors. Proc Natl Acad Sci U S A. 1998;95:14717-22.

7. Inadera H, Hashimoto S, Dong HY, Suzuki T, Nagai S, Yamashita T, et al. WISP-2 as a novel estrogen-responsive gene in human breast cancer cells. Biochem Biophys Res Commun. 2000;275:108-14.

8. Zoubine MN, Banerjee S, Saxena NK, Campbell DR, Banerjee SK. WISP-2: a serum-inducible gene differentially expressed in human normal breast epithelial cells and in MCF-7 breast tumor cells. Biochem Biophys Res Commun. 2001;282:421-5

9. Davies SR, Watkins G, Mansel RE, Jiang WG. Differential expression and prognostic implications of the CCN family members WISP-1, WISP-2, and WISP-3 in human breast cancer. Ann Surg Oncol. 2007;14:1909-18.

10. Banerjee S, Dhar G, Haque I, Kambhampati S, Mehta S, Sengupta K, et al. CCN5/WISP-2 expression in breast adenocarcinoma is associated with less frequent progression of the disease and suppresses the invasive phenotypes of tumor cells. Cancer Res. 2008;68:7606-12. 
11. Haque I, Banerjee S, De A, Maity G, Sarkar S, Majumdar M, et al. CCN5/MISP2 promotes growth arrest of triple-negative breast cancer cells through accumulation and trafficking of p27(Kip1) via Skp2 and FOXO3a regulation. Oncogene. 2015;34:3152-63.

12. Fritah A, Saucier C, De Wever O, Bracke M, Bieche I, Lidereau R, et al. Role of WISP-2/CCN5 in the maintenance of a differentiated and noninvasive phenotype in human breast cancer cells. Mol Cell Biol. 2008;28:1114-23.

13. Ji J, Jia S, Jia Y, Ji K, Hargest R, Jiang WG. WISP-2 in human gastric cancer and its potential metastatic suppressor role in gastric cancer cells mediated by JNK and PLC-gamma pathways. Br J Cancer. 2015;113:921-33.

14. Li L, Cui Y, Ji JF, Jiang WG. Clinical correlation between WISP2 and betacatenin in gastric Cancer. Anticancer Res. 2017;37:4469-73.

15. Dhar G, Mehta S, Banerjee S, Gardner A, McCarty BM, Mathur SC, et al. Loss of WISP-2/CCN5 signaling in human pancreatic cancer: a potential mechanism for epithelial-mesenchymal-transition. Cancer Lett. 2007;254:63-70.

16. Yang Q, Zhang F, Ding Y, Huang J, Chen S, Wu Q, et al. Antitumour activity of the recombination polypeptide GST-NT21MP is mediated by inhibition of CXCR4 pathway in breast cancer. Br J Cancer. 2014;110:1288-97.

17. Tao YS, Ma XY, Chai DM, Ma L, Feng ZZ, Cheng ZN, et al. Overexpression of MMP-1 and VEGF-C is associated with a less favorable prognosis in esophageal squamous cell carcinoma. Onkologie. 2012;35:651-6.

18. Xia J, Cheng L, Mei C, Ma J, Shi Y, Zeng F, et al. Genistein inhibits cell growth and invasion through regulation of miR-27a in pancreatic cancer cells. Curr Pharm Des. 2014;20:5348-53.

19. Chien CS, Ma KH, Lee HS, Liu PS, Li YH, Huang YS, et al. Dual effect of capsaicin on cell death in human osteosarcoma G292 cells. Eur J Pharmacol. 2013;718:350-60

20. Cheng XS, Zhao KP, Jiang X, Du LL, Li XH, Ma ZW, et al. Nmnat2 attenuates tau phosphorylation through activation of PP2A. Journal of Alzheimer's disease : JAD. 2013;36:185-95.

21. Du LL, Chai DM, Zhao LN, Li XH, Zhang FC, Zhang HB, et al. AMPK activation ameliorates Alzheimer's disease-like pathology and spatial memory impairment in a streptozotocin-induced Alzheimer's disease model in rats. Journal of Alzheimer's disease : JAD. 2015;43:775-84.

22. Ma J, Zeng F, Ma C, Pang H, Fang B, Lian C, et al. Synergistic reversal effect of epithelial-to-mesenchymal transition by miR-223 inhibitor and genistein in gemcitabine-resistant pancreatic cancer cells. Am J Cancer Res. 2016;6:1384-95.

23. Kleer CG. Dual roles of CCN proteins in breast cancer progression. J Cell Commun Signal. 2016;10:217-22.

24. Li J, Ye L, Owen S, Weeks HP, Zhang Z, Jiang WG. Emerging role of CCN family proteins in tumorigenesis and cancer metastasis (review). Int J Mol Med. 2015;36:1451-63.

25. Noman MZ, Janji B, Abdou A, Hasmim M, Terry S, Tan TZ, et al. The immune checkpoint ligand PD-L1 is upregulated in EMT-activated human breast cancer cells by a mechanism involving ZEB-1 and miR-200. Oncoimmunology. 2017;6:e1263412.

26. Ferrand N, Gnanapragasam A, Dorothee G, Redeuilh G, Larsen AK, Sabbah M. Loss of WISP2/CCN5 in estrogen-dependent MCF7 human breast cancer cells promotes a stem-like cell phenotype. PLoS One. 2014;9:e87878.

27. Chen Z, Tang J, Cai X, Huang Y, Gao Q, Liang L, et al. HBx mutations promote hepatoma cell migration through the Wnt/beta-catenin signaling pathway. Cancer Sci. 2016;107:1380-9.

28. Tomimaru Y, Koga H, Yano H, de la Monte S, Wands JR, Kim M. Upregulation of T-cell factor-4 isoform-responsive target genes in hepatocellular carcinoma. Liver Int. 2013;33:1100-12.

29. Wong SHM, Fang CM, Chuah LH, Leong CO, Ngai SC. E-cadherin: its dysregulation in carcinogenesis and clinical implications. Crit Rev Oncol Hematol. 2018;121:11-22.

30. Prieto-Garcia E, Diaz-Garcia CV, Garcia-Ruiz I, Agullo-Ortuno MT. Epithelial-tomesenchymal transition in tumor progression. Med Oncol. 2017;34:122.

Ready to submit your research? Choose BMC and benefit from:

- fast, convenient online submission

- thorough peer review by experienced researchers in your field

- rapid publication on acceptance

- support for research data, including large and complex data types

- gold Open Access which fosters wider collaboration and increased citations

- maximum visibility for your research: over $100 \mathrm{M}$ website views per year

At BMC, research is always in progress.

Learn more biomedcentral.com/submissions 